\title{
Fatty acid binding proteins 4 and 5 in overweight prepubertal boys: effect of nutritional counselling and supplementation with an encapsulated fruit and vegetable juice concentrate
}

\author{
Jose A. Canas ${ }^{1}$, L. Damaso ${ }^{1}$, J. Hossain ${ }^{2}$ and P. Babu Balagopal ${ }^{3}$ \\ ${ }^{1}$ Pediatric Endocrinology and Metabolism, Nemours Children's Specialty Care, Jacksonville, FL 32207, USA \\ ${ }^{2}$ Bioinformatics Core Facility, Nemours Children's Specialty Care, Jacksonville, FL 32207, US A \\ ${ }^{3}$ Biomedical Research, Nemours Children's Specialty Care, Jacksonville, FL 32207, USA
}

(Received 22 August 2015 - Final revision received 25 September 2015 - Accepted 5 October 2015)

Journal of Nutritional Science (2015), vol. 4, e39, page 1 of 8

doi:10.1017/jns.2015.29

Abstract

Elevated fatty acid binding proteins (FABP) may play a role in obesity and co-morbidities. The role of nutritional interventions in modulating these levels remains unclear. The aim of this post hoc study was to determine the effect of overweight (OW) on FABP4 and FABP5 in boys in relation to indices of adiposity, insulin resistance and inflammation, and to investigate the effects of a 6-month supplementation with an encapsulated fruit and vegetable juice concentrate (FVJC) plus nutritional counselling (NC) on FABP levels. A post hoc analysis of a double-blind, randomised, placebo-controlled study of children recruited from the general paediatric population was performed. A total of thirty age-matched prepubertal boys (nine lean and twenty-one OW; aged 6-10 years) were studied. Patients received NC by a registered dietitian and were randomised to FVJC or placebo capsules for 6 months. FABP4, FABP5, glucose, insulin, homeostasis model assessment-insulin resistance (HOMA-IR), glucose-induced acute insulin response (AIR), lipidcorrected $\beta$-carotene (LC $\beta C$ ), adiponectin, leptin, high-sensitivity C-reactive protein (hs-CRP), IL- 6 and body composition by dual-energy X-ray absorptiometry were determined before and after the intervention. FABP were higher $(P<0.01)$ in the OW $v$. lean boys and correlated directly with HOMA-IR, abdominal fat mass (AFM), hs-CRP, IL-6, and LC $\beta C(P<0.05$ for all). FABP4 was associated with adiponectin and AIR $(P<0 \cdot 05)$. FVJC plus NC reduced FABP4, HOMA-IR and AFM $(P<0.05$ for all $)$ but not FABP5. OW boys showed elevated FABP4 and FABP5, but only FABP4 was lowered by the FVJC supplement.

Key words: Obesity: Children: Fatty acid binding proteins: $\beta$-Carotene

Childhood nutrition plays a significant role in the management of obesity and related co-morbidities such as CVD and type 2 diabetes mellitus. Alterations and/or deficiencies in lipophilic micronutrients may contribute to the excess accumulation of intra-abdominal adipose tissue and dysregulation of carbohydrate and lipid metabolism ${ }^{(1,2)}$. Fatty acid binding proteins (FABP) are members of a highly conserved tissue-specific family of cytosolic lipid chaperones expressed in adipocytes and macrophages ${ }^{(3)}$ that may contribute to the regulation of energy metabolism, insulin resistance and inflammation by activating nuclear receptors such as retinoic acid receptors and $\mathrm{PPAR}^{(4)}$. FABP are also implicated in the cellular uptake and transport of lipophilic micronutrients that integrate metabolic and inflammatory responses involved in obesity-related diseases $^{(5,6)}$. In animal models, FABP4 deficiency protects against the development of insulin resistance, inflammation, diabetes and atherosclerosis in both genetic and dietary forms of obesity ${ }^{(7,8)}$. In adult humans, decreased FABP4 expression is associated with lower TAG and reduced risk for CVD and type 2 diabetes mellitus ${ }^{(9)}$. While elevated levels

Abbreviations: AIR, acute insulin response; FABP, fatty acid binding protein; FVJC, fruit and vegetable juice concentrate; NC, nutritional counselling; OW, overweight

* Corresponding author: J. Atilio Canas, email jcanas@nemours.org 
of FABP4 have also been reported both in obese children and adults, its relationship with obesity-related risk factors for CVD remains less clear ${ }^{(10-16)}$. Children with lower FABP4 concentrations who consume high-fat diets do not show a concomitant increase in fasting insulin or high-sensitivity C-reactive protein, whereas those with higher FABP4 concentrations show marked increases in these measures, which correlate with the intake of saturated fat ${ }^{(13)}$. In adults, FABP4 has been shown to correlate directly with the glucose-induced acute insulin response (AIR), and is postulated to play an integral role in coupling $\beta$-cell function with adiposity ${ }^{(17)}$. A few studies have also reported the reversal of the elevated FABP4 levels after weight reduction in children ${ }^{(12,18)}$. Because FABP play important roles and contribute to obesity-related comorbidities, developing nutritional strategies to reduce their levels is important.

The primary objectives of this post hoc analysis performed on a double-blind placebo-controlled study was to determine the effect of overweight (OW) on FABP4 and FABP5 and the effect of nutritional counselling (NC) and supplementation with fruit and vegetable juice concentrate (FVJC) on these levels in lean and OW boys.

\section{Experimental methods}

\section{Trial procedures}

This study was conducted in accordance with the guidelines laid down in the Declaration of Helsinki, and all procedures involving human subjects/patients were approved by the Institutional Review Committee at Wolfson Children's Hospital, Jacksonville, FL. Written parental informed consent and child's assent were obtained for all participants upon enrolment in the study. Subjects received a modest monetary compensation for their participation in the study. The study was registered at http://www.clinicaltrials.gov (NCT00842543).

\section{Participants}

Detailed description of the subjects as well as data on certain cardiometabolic risk factors in the same subjects have been previously reported ${ }^{(19)}$. Briefly, this was a post hoc analysis performed on a total of thirty-nine prepubertal boys (age 6-10 years) enrolled, thirty completers (nine lean with $\mathrm{BMI} \leq 85$ th percentile and twenty-one $\mathrm{OW}$ with $\mathrm{BMI}>85$ th percentile) and nine dropouts (due to fear of the second blood draw).

Subjects with a history of chronic illness or chronic medications were excluded from the study. Those with illness or bone fracture within 2 weeks of their blood draw were also excluded. The participants were instructed not to consume any medications, including vitamins, herbal remedies or anti-inflammatory drugs within $30 \mathrm{~d}$ of the anticipated blood draw.

\section{Randomisation}

The intent-to-treat principle was applied to thirty-nine subjects who were randomised utilising a randomisation scheme generated by the website Randomization.com (http://www. randomization.com) and who received either active supplement or identical placebo capsules provided by the manufacturer (Juice Plus $+\circledR$; NSA, LLC) in conjunction with 6 months of NC.

\section{Design}

The detailed study protocol and design have been previously described $^{(19)}$. A modified rapid intravenous glucose tolerance test was performed as previously described ${ }^{(20)}$. All subjects received a $60-\mathrm{min}$ NC session with a registered dietitian. Instructions emphasised to limit saturated fats to $<7 \%$ of total energy intake, with a minimum of five servings of fruits and vegetables per $\mathrm{d}$, and moderate daily activity was encouraged. Instructions were re-emphasised after 3 months. Subjects were instructed to take one study capsule with breakfast and dinner. Two active capsules of the supplement provide approximately $3.75 \mathrm{mg} \beta$-carotene, $117 \mathrm{mg}$ of vitamin C, $22.5 \mathrm{IU}$ of vitamin $\mathrm{E}$ (23 $\mathrm{mg} \alpha$-tocopherol equivalents), $210 \mu \mathrm{g}$ folate, $30 \mathrm{mg} \mathrm{Ca}$ and $21 \mathrm{~kJ}$ per $\mathrm{d}$. The orchard concentrate consists of $850 \mathrm{mg}$ of dried powder blend of juice and pulp $(52 \%)$ with apple, orange, pineapple, cranberry, peach, acerola cherry and papaya in varying proportions, in addition to beetroot powder, date fibre and prune fibre. The garden blend contains $750 \mathrm{mg}$ of dried powder blend of juice and pulp $(60 \%)$ of carrot, parsley, beet, kale, broccoli, cabbage, tomato and spinach, in varying proportions, as well as sugar beet fibre, garlic powder, oat bran fibre and rice bran. Moreover, each powder was enriched with vitamins (vitamin $\mathrm{C}$, vitamin $\mathrm{E}$ and folic acid) and carotenoids ( $\beta$-carotene). These supplements are commercial products that have been recently chemically characterised by an independent group. Within the orchard blend, twenty-eight compounds with a predominance of flavonols have been identified. A total of twenty-five compounds, belonging to different phenolic classes have been identified within the garden blend ${ }^{(21)}$.

\section{Assays}

Blood was collected after $10 \mathrm{~h}$ of fasting and carefully processed under orange lights immediately after collection; serum and plasma were aliquoted and frozen in opaque tubes at $-80^{\circ} \mathrm{C}$ until analysis. FABP4 (Cayman Chemical; interassay CV $3.9 \%$ ) and FABP5 (BioVendor; interassay CV $5.8 \%$ ) were measured by ELISA. The following measurements were also made as previously described ${ }^{(19)}$. $\beta$-Carotene was measured by HPLC with photodiode array detection, glucose was measured using an Analox GM7 Glucose Analyser (Analox Instruments; interassay $\mathrm{CV}<5 \%$ ) and insulin was measured by a commercially available RIA kit (EMD Millipore Life Sciences; interassay CV $<10 \%$ ). Total cholesterol, TAG and HDL-cholesterol concentrations were measured using colorimetric assays (Beckman DxC 800 Analyzer; Beckman Coulter, Inc.; interassay $\mathrm{CV}<2 \cdot 6,<2$, and $<3 \%$, respectively), IL-6 by specific ELISA (R\&D Systems; interassay CV $7 \cdot 8 \%$ ), high-sensitivity C-reactive protein by immuno-nephelometry (Siemens Healthcare Diagnostics; interassay $\mathrm{CV}<5 \%$ ) and adiponectin and leptin by RIA 
Table 1. Baseline clinical and biochemical characteristics of study subjects by weight group (Mean values and standard deviations; medians and interquartile ranges (IQR))

\begin{tabular}{|c|c|c|c|c|c|}
\hline & \multicolumn{2}{|c|}{ Lean $(n 13)$} & \multicolumn{2}{|c|}{ Overweight ( $n$ 26) } & \multirow{2}{*}{$P^{\star}$} \\
\hline & Median & IQR & Median & IQR & \\
\hline Age (years) & & & & & NS \\
\hline Mean & $9 \cdot 36$ & & 9.04 & & \\
\hline SD & 1.31 & & 1.43 & & \\
\hline BMI Z-score & & & & & $<0.05$ \\
\hline Mean & -0.08 & & $2 \cdot 14$ & & \\
\hline SD & 1.43 & & 0.47 & & \\
\hline Abdominal fat mass $(\mathrm{kg})$ & & & & & $<0.05$ \\
\hline Mean & 1.249 & & 4.144 & & \\
\hline $\mathrm{SD}$ & 0.391 & & 0.141 & & \\
\hline $\mathrm{LC} \beta \mathrm{C}(\mu \mathrm{mol} / \mathrm{l})$ & 0.055 & $0.038-0.093$ & 0.035 & $0.023-0.066$ & 0.04 \\
\hline FABP4 $(\mathrm{ng} / \mathrm{ml}) \dagger$ & 9.92 & $8 \cdot 5-11.4$ & $30 \cdot 67$ & $26 \cdot 7-34 \cdot 6$ & $<0.001$ \\
\hline FABP5 $(\mathrm{ng} / \mathrm{ml}) \dagger$ & 3.33 & $2.8-3.9$ & 6.76 & $5 \cdot 7-7.9$ & $<0.001$ \\
\hline TAG $(\mathrm{mmol} / \mathrm{l})$ & 0.52 & $0.37-0.75$ & 0.96 & $0.61-1.20$ & 0.004 \\
\hline HOMA-IR & 1.34 & $0.8-1.82$ & 3.68 & $1.98-4.01$ & $<0.001$ \\
\hline AIR & 345 & $206-597$ & 687 & $402-1,014$ & 0.014 \\
\hline GDI & & & & & NS \\
\hline Mean & 1.67 & & 1.50 & & \\
\hline SD & 0.30 & & 0.25 & & \\
\hline hs-CRP (mg/l) & 0.36 & $0.16-0.39$ & 0.93 & $0.38-0.93$ & 0.01 \\
\hline IL-6 (pg/ml) & 0.69 & $0.64-1.07$ & 1.35 & $0.92-2.3$ & 0.018 \\
\hline Leptin (ng/ml) & 3.76 & $2 \cdot 5-4.8$ & $21 \cdot 3$ & $10 \cdot 6-26 \cdot 5$ & $<0.001$ \\
\hline Adiponectin $(\mu \mathrm{g} / \mathrm{ml})$ & 14.7 & $10 \cdot 8-20.9$ & $10 \cdot 7$ & $7 \cdot 6-12 \cdot 4$ & 0.04 \\
\hline Leptin:adiponectin ratio & 0.23 & $0.19-0.31$ & 1.85 & $1.02-2.67$ & $<0.001$ \\
\hline
\end{tabular}

LC $\beta C$, lipid-corrected $\beta$-carotene; FABP, fatty acid binding protein; HOMA-IR, homeostasis model assessment-insulin resistance; AIR, acute insulin response; GDI, glucose disposal index; hs-CRP, high-sensitivity C-reactive protein.

* $P$ values represent differences between groups as determined by the independent two-sample $t$ test or Mann-Whitney test for continuous variables.

† FABP4 and FABP5 data have not been previously reported ${ }^{(19)}$.

(EMD Millipore Life Sciences; interassay CV 8.5 and $4.5 \%$, respectively).

\section{Calculations}

The homeostasis model assessment-insulin resistance (HOMAIR) was calculated using the following formula: fasting glucose $(\mathrm{mmol} / \mathrm{l}) \times$ fasting insulin $(\mu \mathrm{U} / \mathrm{ml}) / 22 \cdot 5$. AIR was defined as the mean incremental rise in plasma insulin at 3 and $5 \mathrm{~min}$ after a rapid intravenous load of glucose. To adjust AIR for the effects of insulin sensitivity, a glucose disposal index was calculated as $\log _{10}$ (AIR $\times$ fasting glucose concentration/fasting insulin concentration) in a manner similar to the correction of AIR using the insulin sensitivity index that is used in the minimal model intravenous glucose tolerance test ${ }^{(22)}$.

$\beta$-Carotene concentrations are closely correlated with major lipid distribution and were corrected for lipid status (lipidcorrected $\beta$-carotene) by dividing by the sum of total cholesterol and TAG expressed in $\mathrm{mmol} / \mathrm{l}^{(23)}$. The leptin:adiponectin ratio was also calculated.

\section{Statistical analyses}

The intention-to-treat principle was applied to all patients included in the primary analysis. In the post hoc analysis, quantitative variables are presented using either mean with standard deviation or in the case of substantially skewed distribution, median and interquartile range. Categorical variables are presented using frequencies and percentages. The two-sample $t$ test or a non-parametric Mann-Whitney $U$ test, whichever was appropriate, was used to compare quantitative variables. The Wilcoxon signed-rank test was used for paired sample comparisons of non-parametric variables.

A multivariate ANOVA for repeated measures was performed to compare the mean changes from baseline at the 3 -month and 6-month visits for the variables of interest. The least squared means with standard errors and $P$ values for both lean and obese subjects are presented at 3 and 6 months in Table 2. Both models were adjusted for baseline values of the corresponding variable and the percentage change in weight. Associations between FABP4 and FABP5 with other variables were examined with unadjusted univariate analysis followed by multivariate linear regression analysis, adjusting for potential confounders to determine independent predictors of plasma FABP4 and FABP5. All tests were twotailed at the level of significance of $\leq 0 \cdot 05$. The statistical software SPSS version 22.0 (SPSS) was used for analyses.

\section{Results}

\section{Participant characteristics}

The clinical and biochemical characteristics of the study participants by BMI (Table 1) and treatment group are presented in Table 2. Although subject characteristics and some biological factors have been published previously ${ }^{(19)}$, for clarity of discussion, we have included some of the data pertinent to the present study along with new data on FABP4 and FABP5.

Subjects were classified lean if BMI was $\leq 85$ th percentile and $\mathrm{OW}$ if $\mathrm{BMI}$ was $>85$ th percentile. By design, significant 
Table 2. Mixed-model analysis of the changes in concentrations between fruit and vegetable juice concentrate (FVJC) and placebo in the overweight group ${ }^{\star}$ (Least squared means (LS means) with their standard errors)

\begin{tabular}{|c|c|c|c|c|c|c|c|c|c|c|c|}
\hline & \multicolumn{4}{|c|}{ FVJC $(n 11)$} & \multicolumn{4}{|c|}{ Placebo ( $n$ 10) } & & & \\
\hline & \multicolumn{2}{|c|}{0 months } & \multicolumn{2}{|c|}{6 months } & \multicolumn{2}{|c|}{0 months } & \multicolumn{2}{|c|}{6 months } & \multicolumn{2}{|c|}{ Mean difference } & \multirow[b]{2}{*}{$P$} \\
\hline & LS mean & $\mathrm{SE}$ & LS mean & SE & LS mean & SE & LS mean & $\mathrm{SE}$ & LS mean & SE & \\
\hline $\log L C \beta C$ & -0.66 & 0.07 & -0.14 & 0.1 & -0.78 & 0.07 & -0.93 & 0.11 & -1.07 & 0.1 & $<0.001$ \\
\hline Log FABP4 & 1.41 & 0.04 & 1.37 & 0.03 & 1.52 & 0.05 & 1.49 & 0.37 & -0.13 & 0.05 & 0.025 \\
\hline Log FABP5 & 0.7 & 0.06 & 0.7 & 0.05 & 0.74 & 0.06 & 0.76 & 0.05 & -0.01 & 0.06 & 0.9 \\
\hline Log HOMA-IR† & 1.27 & 0.1 & 1.2 & 0.1 & 1.34 & 0.1 & 1.43 & 0.1 & -0.23 & 0.1 & 0.014 \\
\hline Log leptin & 1.22 & 0.06 & 1.2 & 0.08 & 1.26 & 0.06 & 1.34 & 0.06 & 0.54 & 0.03 & 0.297 \\
\hline Log adiponectin & 3.95 & 0.05 & 4.07 & 0.05 & 4.04 & 0.05 & 4.04 & 0.06 & 0.03 & 0.02 & 0.192 \\
\hline
\end{tabular}

LC $\beta C$, lipid-corrected $\beta$-carotene; FABP, fatty acid binding protein; HOMA-IR, homeostasis model assessment-insulin resistance.

* Adjusted for percentage change in body weight.

† Log HOMA-IR data have been previously reported ${ }^{(19)}$.

differences between the lean and OW groups exist at baseline in terms of adiposity including BMI $Z$-score and abdominal fat mass $(P \leq 0 \cdot 05$; Table 1). Pill count data were available for 88 $\%$ of all supplement bottles dispensed. At 3 months, $60 \%$ of the lean and $55 \%$ of the OW subjects had taken $>75 \%$ of the active supplement capsules, and at 6 months, $80 \%$ of the lean and $50 \%$ of the OW children had $>75 \%$ compliance. However, the groups were not statistically different.

\section{Correlations}

FABP4 and FABP5 were higher in the OW $v$. lean boys (Table 1) and directly correlated with abdominal fat mass and leptin:adiponectin ratio at baseline and throughout the study (Fig. 1(a)-(d)); FABP4 $(P=0.002)$ and FABP5 $(P=$ $0 \cdot 007)$ were also directly correlated with $\log$ HOMA-IR at baseline, but FABP5 lost its correlation at 3 months postintervention $(P=0.265)$ (Fig. 1(e) and (f)). FABP4 $(r 0.823$; $P \leq 0.001)$ and FABP5 $(r 0.699 ; P \leq 0.001)$ were directly correlated with leptin. FABP4 $(r-0.439 ; P=0.015)$ was negatively correlated with adiponectin at baseline and maintained its correlation throughout the study time points. FABP5 $(r$ $-0.370 ; P=0.044)$ was negatively correlated with adiponectin at baseline, but lost its correlation at the 3-and 6-month time points (data not shown). In contrast, FABP4 $(P=0 \cdot 006)$ and FABP5 $(P=0.011)$ had a negative correlation with lipidcorrected $\beta$-carotene at baseline, but FABP5 lost its correlation at 3 months (Fig. $1(\mathrm{~g})$ and (h)). FABP4 $(P=0.014)$ and FABP5 $(P=0.005)$ were directly correlated with highsensitivity C-reactive protein, but only FABP5 was directly correlated with IL-6 $(P=0.015)$ at baseline. In multiple regression analyses, BMI $Z$-score explained $66 \%$ of the variance for FABP 4 and $47 \%$ of the variance for FABP5. When adiponectin was added to the model, $75 \%$ of the variance was explained by FABP4 and $49 \%$ by FABP5 $(P<0.001)$ for all. No collinearity violations were observed between variables.

\section{Treatment effects}

FVJC supplementation substantially reduced FABP4 $(P=$ $0 \cdot 025)$ when corrected for percentage change in abdominal fat mass, as opposed to FABP5, which showed no significant treatment effect $(P=0.231)$ when compared with placebo (Table 2 and Fig. 2). Wilcoxon signed-ranks tests determined inverse correlations between the percentage change in log lipid-corrected $\beta$-carotene $(Z=-2.77 ; \quad P=0.005)$ and FABP4 $(Z=-2.128 ; \quad P=0.033)$ but not for FABP5 $(Z=-0.896 ; P=0.370)$.

There were no significant treatment effects between groups for BMI $Z$-score or total body fat. There was a significant treatment effect on the percentage change in abdominal fat mass $(\mathrm{kg})$ measured by dual-energy X-ray absorptiometry for the entire cohort at 6 months, showing that the placebo group had a $11.2(95 \%$ CI 4.16, 18.23$) \%$ increase, as opposed to a $1.47(\mathrm{CI}-8.31,5.37) \%$ decrease in the supplement group $(P=0.029)$.

\section{Discussion}

In this post hoc analysis study, we report not only the effects of childhood adiposity and related metabolic alterations on FABP4 and FABP5 in prepubertal boys but also the outcome of a 6-month intervention with a FVJC supplement on the concentration of the FABP. The data demonstrated higher concentrations of both FABP4 and FABP5 in the OW when compared with the normal-weight boys. While both FABP4 and FABP5 were closely associated with insulin resistance and measures of adiposity, only FABP4 showed a decrease in concentration in response to the 6-month intervention with the FVJC supplement.

The higher levels of FABP4 in OW boys when compared with the levels of their lean counterparts that were observed in our study are similar to previous reports in children ${ }^{(12,13,15,16,18)}$ and adults ${ }^{(3,9-11,14,17,24)}$, but the increased concentration of FABP5 has not been previously reported. Both FABP4 and FABP5 are altered in childhood obesity and showed direct associations with markers of insulin resistance including AIR and HOMA-IR, as well as the leptin:adiponectin ratio, which also has been suggested as an indicator of insulin resistance ${ }^{(25)}$. Our data in non-diabetic prepubertal boys agree with previous observations in adults that FABP4 plays an important role in the $\mathrm{AIR}^{(17)}$. Further, the strong correlations between both FABP and abdominal adiposity observed in the present study suggest that FABP may contribute to the obesity phenotype in boys. 
(a)

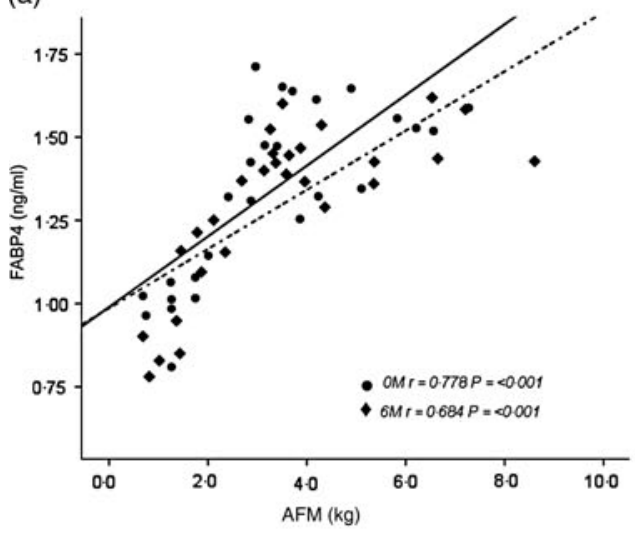

(c)

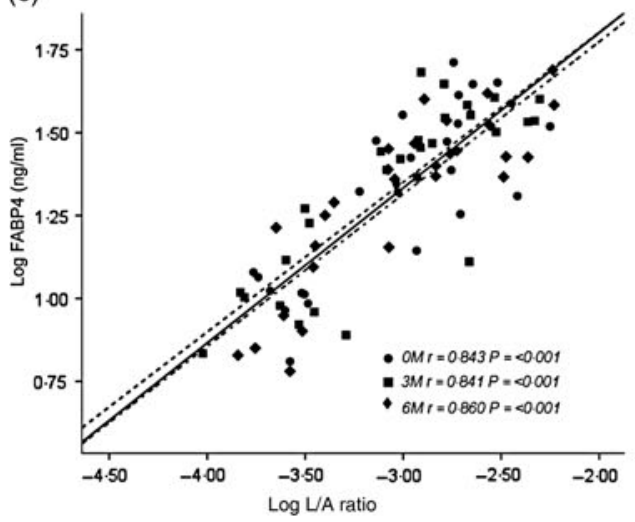

(e)

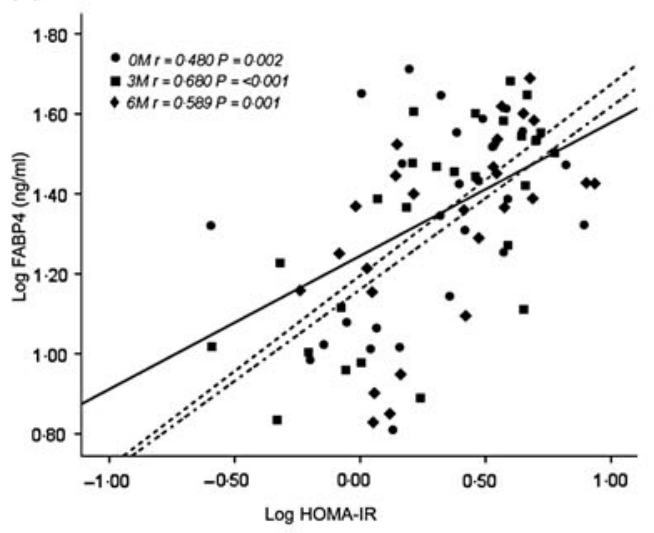

(g)

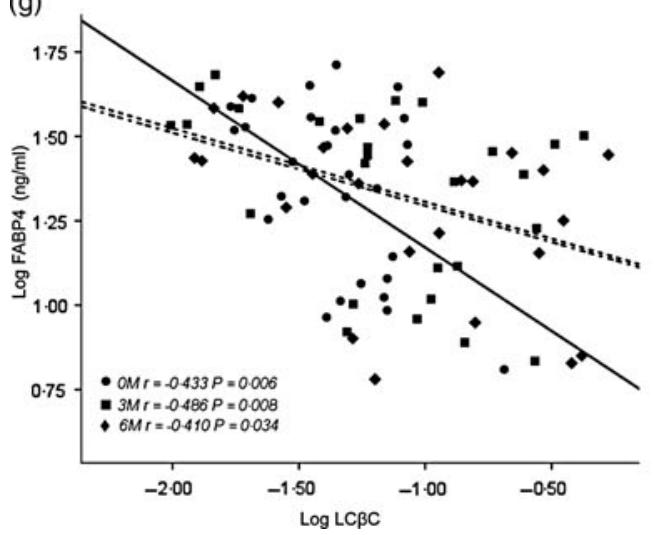

(b)

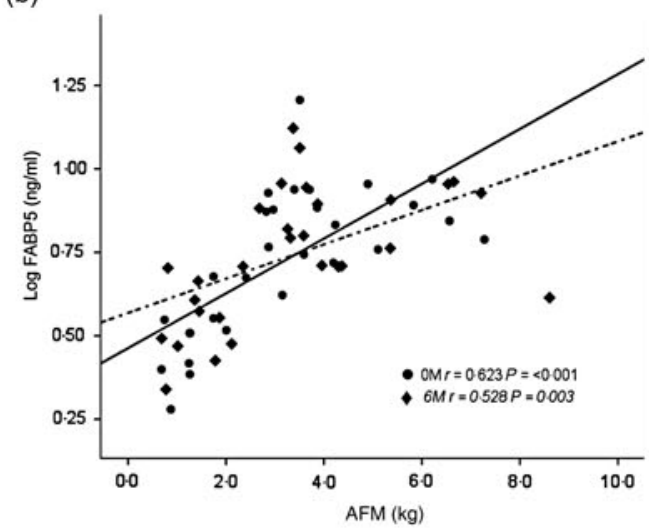

(d)

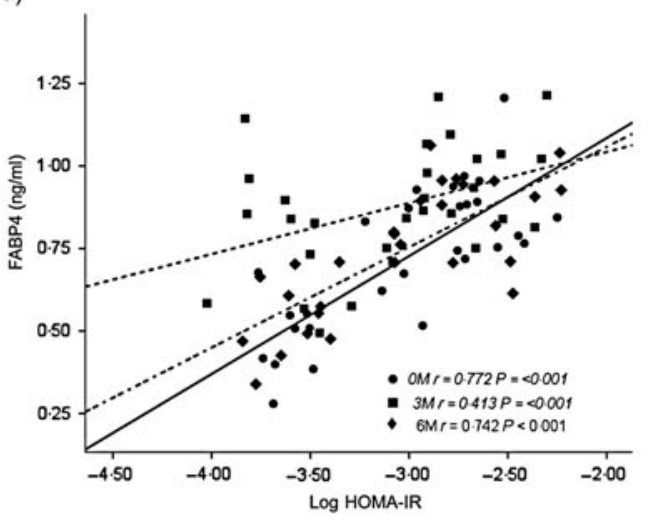

(f)

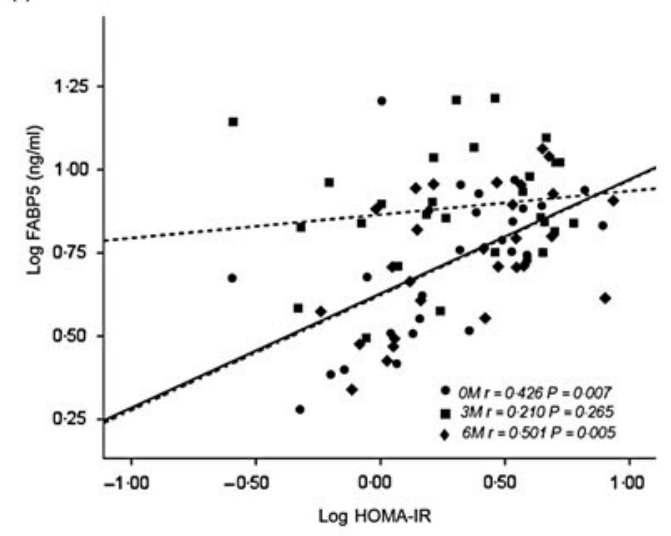

(h)

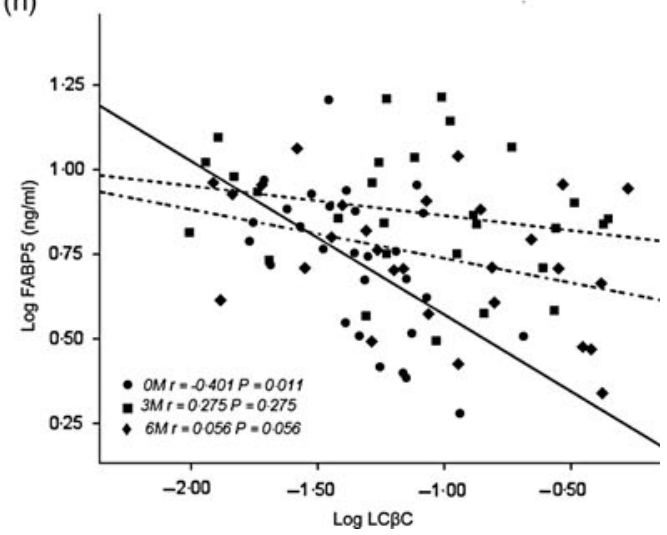

Fig. 1. Pearson's correlations between fatty acid binding protein (FABP) 4 and FABP5 and abdominal fat mass (AFM) in kg (a, b), leptin:adiponectin ratio (L/A) (c, d), homeostasis model assessment-insulin resistance (HOMA-IR) $(\mathrm{e}, \mathrm{f})$ and lipid-corrected $\beta$-carotene $(\mathrm{LC} \beta \mathrm{C})(\mathrm{g}, \mathrm{h})$ at baseline $(-; 0 \mathrm{M} ; \mathbf{\bullet}), 3 \mathrm{months}(----; 3 \mathrm{M} ; \mathbf{\square})$ and 6 months $(-.-; 6 \mathrm{M} ; \bullet)$. 
(a)

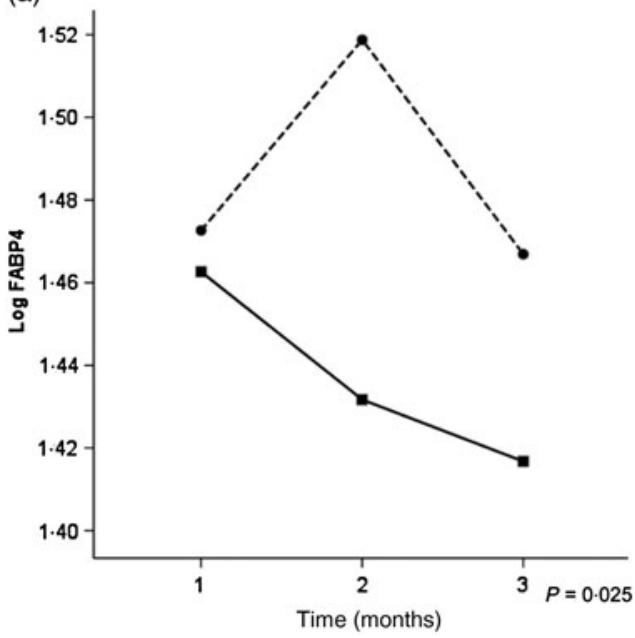

(b)

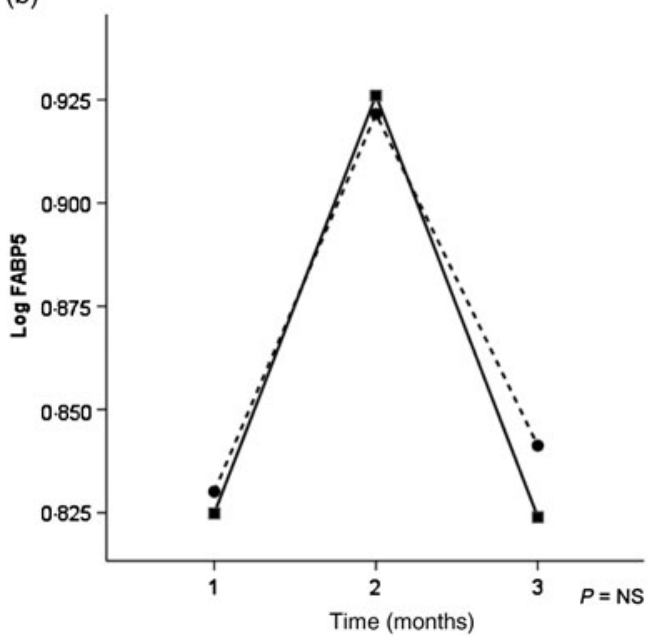

Fig. 2. Fatty acid binding protein (FABP) 4 (a) and FABP5 (b) changes over time (months) between fruit and vegetable juice concentrate-supplemented boys (- -) and those receiving placebo (----) in the overweight group. Values are least squared means.

$\beta$-Carotene is the main carotenoid in human sera and has been proposed as a useful indicator of fruit and vegetable consumption ${ }^{(26,27)}$. While there was an inverse relationship between both FABP and baseline $\beta$-carotene concentration, only the magnitude of decrease in FABP4, and the corresponding increase in $\beta$-carotene concentration were correlated. The FVJC consumption resulted in a significant increase in $\beta$-carotene and a decrease in abdominal fat mass, but only the concentration of FABP4 and not FABP5 was reduced. FABP4 is highly expressed in adipocytes, whereas FABP5 is predominantly epidermal and constitutes only a minor fraction of FABP in the adipocytes (the amount being almost 100-fold lower than that of FABP4) ${ }^{(28)}$. Although metabolically complementary to FABP4, FABP5 did not respond to the FVJC supplementation in a similar fashion, suggesting that the two FABP have a differential response to an external stimulus such as polyphenolic supplementation. It is not known if a similar pattern of response holds for other interventions such as exercise and/or other nutritional/pharmacological therapies.

The underlying mechanisms leading to the specific reduction in FABP4 levels in response to the FVJC supplementation are not apparent from the present study. Responses to dietary supplementation with these compounds can be quite variable between individuals ${ }^{(29)}$ and may have influenced the results of this study. Genome-wide studies have associated common genetic polymorphisms in the $\beta$-carotene oxygenase 1 (BCO1) gene with the obese phenotype in African-American adults ${ }^{(30)}$. BCO1 is the gene that encodes an enzyme that is expressed in the intestine and converts provitamin A carotenoids to retinaldehyde and all-trans-retinoic acid; both can stimulate retinoic acid receptors and reduce PPAR $\gamma$ activity. The reduction in PPAR $\gamma$ activity may lead to a decrease in the expression of PPAR $\gamma$ target genes such as FABP4 with subsequent decreases in adipocyte lipid content, as has been previously reported in mature adipocytes ${ }^{(31)}$ and mouse models $^{(2,32,33)}$. Other anti-adipogenic cooperative or alternative mechanisms may involve flavonoid-mediated inhibition of
Akt activation and GSK3 3 phosphorylation, which induces down-regulation of lipid accumulation and lipid-metabolising gene expression, ultimately inhibiting adipocyte differentiation ${ }^{(34,35)}$. Mice with deletion of both FABP4 and FABP5 have strong protection from diet-induced obesity, insulin resistance, type 2 diabetes and fatty liver disease ${ }^{(5)}$. A smallmolecule dual inhibitor of FABP4/5, when chronically administered to diet-induced obese mice, was recently reported to reduce plasma TAG and NEFA levels, without inducing significant changes in insulin sensitivity ${ }^{(36)}$. Pharmacological agents that modify FABP function may provide tissue-specific or cell-type-specific control of lipid signalling pathways, inflammatory responses and metabolic regulation, potentially providing a new class of drugs for diseases such as obesity, diabetes and atherosclerosis ${ }^{(37)}$. Emerging data from human studies suggest that inhibiting the function of FABP4 may be a potential mechanism for the prevention of metabolic diseases like type 2 diabetes and atherosclerosis ${ }^{(37,38)}$. In the Nurses' Health Study and the Health Professionals Followup Study, a functional variant T87C polymorphism in the FABP4 promoter resulted in reduced adipose FABP4 messenger ribonucleic acid expression and was associated with reduced risk for metabolic dyslipidaemia, type 2 diabetes and coronary atherosclerosis $^{(9)}$. In this context, the lowering of FABP4 in our study via low-dose supplementation with FVJC and NC is important. Using the Penn Diabetes Heart Study, cross-sectional analysis of FABP4 and FABP5 levels had an additive, independent association with the metabolic syndrome and inflammatory markers, but only FABP4 showed a specific association with the presence of coronary artery $\mathrm{Ca}^{(39)}$. Previous studies based on exercise interventions in obese children and adults have shown substantial deceases in FABP4 levels ${ }^{(11,35)}$. In obese children undergoing a 1-year weight-reduction programme, changes in FABP4 concentrations correlated significantly with percentage body fat and leptin, but the decrease in FABP4 levels was limited only to those children who lost substantial amounts of weight ${ }^{(12)}$. These results are similar to our study. A larger study in prepubertal children 
also showed that FABP4 was significantly associated with changes in both weight and waist circumference over 3 years of follow-up, and higher concentrations at baseline predicted the development of the metabolic syndrome ${ }^{(16)}$; these results are similar to those reported in adults ${ }^{(40)}$. Another study in children found that FABP4 predicted metabolic syndrome components with almost $68 \%$ accuracy independent of BMI status and that a weight-reduction programme with diet and exercise was effective in reducing FABP4, BMI percentile, waist circumference, TAG and cholesterol ${ }^{(15)}$. The beneficial improvements in FABP4, insulin resistance, TAG levels, abdominal fat, and the differential regulation of FABP with $\mathrm{NC}$ and the FVJC supplementation suggest that carotenoids, along with other polyphenols, may play a role in the regulation of abdominal adiposity, similar to that reported in animal models ${ }^{(32,41)}$, and warrant further exploration in the paediatric population ${ }^{(19,42)}$.

Strengths of the study include the randomised double-blind, placebo-controlled prospective nature of the intervention and the inclusion of both lean and obese subjects. The limitations of the study include the relatively small sample size; lower than expected rates of compliance; lack of inclusion of both sexes in the study cohort; and the use of surrogate markers for insulin resistance, $\beta$-cell function and abdominal adiposity. We also did not measure all-trans-retinoic acid; however, a previous intervention study using carrot juice, which is rich in $\beta$-carotene, reported a doubling of plasma all-trans-retinoic acid levels without significant increase in serum retinol ${ }^{(43)}$. The data were not adjusted for compliance rates because there were no significant differences between groups. Further, $\beta$-carotene represents only one of several polyphenolic compounds present in the supplement ${ }^{(21)}$, and this precludes us from drawing definitive conclusions about ascribing a unique role to $\beta$-carotene as solely responsible for the observed effects in the present study.

In conclusion, in this post hoc analysis, we report higher levels of both FABP4 and FABP5 in OW $v$. lean prepubertal boys and show a reduction in circulating FABP4 along with beneficial improvements in insulin sensitivity and abdominal adiposity in the children supplemented with the FVJC for a sustained period of 6 months. Although the mechanisms remain unclear, given the strong relationship between FABP4 and indicators of adiposity and metabolic dysfunction and the beneficial effect of the supplementation, future studies are warranted to corroborate these findings.

\section{Acknowledgements}

We thank the volunteers and their families for participating in this study. We are grateful to Sheila Smith; the nursing staff at the clinical research centre of Wolfson Children's Hospital for their expert support; and the excellent technical support of Shawn Sweeten, Karl Mann and Astride Altomare of the Nemours Biomedical Analysis Laboratory. We also thank Vicky Funanage, PhD, for her support.

This work was supported by the Nemours Research Program. The placebo and active study capsules were a gift from NSA, LLC, Collierville, TN. NSA had no role in the design, analysis or writing of this article.
J. A. C. has disclosed a financial interest; all other authors have nothing to disclose.

The authors' responsibilities were as follows - J. A. C.: study design, data collection, data analysis and writing of the manuscript; L. D.: study design, data collection and review of the manuscript; J. H.: data analysis; and P. B. B.: study design, data analysis and writing of the manuscript.

\section{References}

1. Landrier J-F, Marcotorchino J \& Tourniaire F (2012) Lipophilic micronutrients and adipose tissue biology. Nutrients 4, 1622-1649.

2. Bonet ML, Canas JA, Ribot J, et al. (2015) Carotenoids and their conversion products in the control of adipocyte function, adiposity and obesity. Arch Biochem Biophys 572, 112-125.

3. Maeda K, Uysal KT, Makowski L, et al. (2003) Role of the fatty acid binding protein mal1 in obesity and insulin resistance. Diabetes $\mathbf{5 2}$, 300-307.

4. Tan NS, Shaw NS, Vinckenbosch N, et al. (2002) Selective cooperation between fatty acid binding proteins and peroxisome proliferator-activated receptors in regulating transcription. Mol Cell Biol 22, 5114-5127.

5. Maeda K, Cao H, Kono K, et al. (2005) Adipocyte/macrophage fatty acid binding proteins control integrated metabolic responses in obesity and diabetes. Cell Metab 1, 107-119.

6. Furuhashi M, Fucho R, Gorgun CZ, et al. (2008) Adipocyte/ macrophage fatty acid-binding proteins contribute to metabolic deterioration through actions in both macrophages and adipocytes in mice. J Clin Invest 118, 2640-2650.

7. Hotamisligil GS, Johnson RS, Distel RJ, et al. (1996) Uncoupling of obesity from insulin resistance through a targeted mutation in aP2, the adipocyte fatty acid binding protein. Science 274, 1377-1379.

8. Uysal KT, Scheja L, Wiesbrock SM, et al. (2000) Improved glucose and lipid metabolism in genetically obese mice lacking aP2. Endocrinology 141, 3388-3396.

9. Tuncman G, Erbay E, Hom X, et al. (2006) A genetic variant at the fatty acid-binding protein aP2 locus reduces the risk for hypertriglyceridemia, type 2 diabetes, and cardiovascular disease. Proc Natl Acad Sci U S A 103, 6970-6975.

10. Xu A, Wang Y, Xu JY, et al. (2006) Adipocyte fatty acid-binding protein is a plasma biomarker closely associated with obesity and metabolic syndrome. Clin Chem 52, 405-413.

11. Mohlig M, Weickert MO, Ghadamgadai E, et al. (2007) Adipocyte fatty acid-binding protein is associated with markers of obesity, but is an unlikely link between obesity, insulin resistance, and hyperandrogenism in polycystic ovary syndrome women. Eur J Endocrinol 157, 195-200.

12. Reinehr T, Stoffel-Wagner B \& Roth CL (2007) Adipocyte fatty acid-binding protein in obese children before and after weight loss. Metabolism 56, 1735-1741.

13. Aeberli I, Beljean N, Lehmann R, et al. (2008) The increase of fatty acid-binding protein aP2 in overweight and obese children: interactions with dietary fat and impact on measures of subclinical inflammation. Int J Obes (Lond) 32, 1513-1520.

14. Makowski L \& Hotamisligil GS (2005) The role of fatty acid binding proteins in metabolic syndrome and atherosclerosis. Curr Opin Lipidol 16, 543-548.

15. Krzystek-Korpacka M, Patryn E, Bednarz-Misa I, et al. (2011) Circulating adipocyte fatty acid-binding protein, juvenile obesity, and metabolic syndrome. J Pediatr Endocrinol Metab 24, 921-928.

16. Choi KM, Yannakoulia M, Park MS, et al. (2011) Serum adipocyte fatty acid-binding protein, retinol-binding protein 4 , and adiponectin concentrations in relation to the development of the metabolic syndrome in Korean boys: a 3-y prospective cohort study. Am J Clin Nutr 93, 19-26.

17. Wu LE, Samocha-Bonet D, Whitworth PT, et al. (2014) Identification of fatty acid binding protein 4 as an adipokine that regulates insulin secretion during obesity. Mol Metab 3, 465-473. 
18. Corripio R, Gonzalez-Clemente JM, Perez-Sanchez J, et al. (2010) Weight loss in prepubertal obese children is associated with a decrease in adipocyte fatty-acid-binding protein without changes in lipocalin-2: a 2-year longitudinal study. Eur J Endocrinol 163, 887-893.

19. Canas JA, Damaso L, Altomare A, et al. (2012) Insulin resistance and adiposity in relation to serum $\beta$-carotene levels. J Pediatr 161, 58-64, e52.

20. Weyer C, Bogardus C, Mott DM, et al. (1999) The natural history of insulin secretory dysfunction and insulin resistance in the pathogenesis of type 2 diabetes mellitus. J Clin Invest 104, 787-794.

21. Bresciani L, Calani L, Cossu M, et al. (2015) (Poly)phenolic characterization of three food supplements containing 36 different fruits, vegetables and berries. PharmaNutrition 3, 11-19.

22. Rosenbaum M, Nonas C, Horlick M, et al. (2004) $\beta$-Cell function and insulin sensitivity in early adolescence: association with body fatness and family history of type 2 diabetes mellitus. I Clin Endocrinol Metab 89, 5469-5476.

23. Bjornson LK, Kayden HJ, Miller E, et al. (1976) The transport of $\alpha$-tocopherol and $\beta$-carotene in human blood. I Lipid Res 17, 343-352.

24. Baessler A, Lamounier-Zepter V, Fenk S, et al. (2014) Adipocyte fatty acid-binding protein levels are associated with left ventricular diastolic dysfunction in morbidly obese subjects. Nutr Diabetes 4, e106.

25. Oda N, Imamura S, Fujita T, et al. (2008) The ratio of leptin to adiponectin can be used as an index of insulin resistance. Metabolism 57, 268-273.

26. Neuhouser ML, Rock CL, Eldridge AL, et al. (2001) Serum concentrations of retinol, $\alpha$-tocopherol and the carotenoids are influenced by diet, race and obesity in a sample of healthy adolescents. $J$ Nutr 131, 2184-2191.

27. Burrows TL, Warren JM, Colyvas K, et al. (2008) Validation of overweight children's fruit and vegetable intake using plasma carotenoids. Obesity 17, 162-168.

28. Coe NR, Simpson MA \& Bernlohr DA (1999) Targeted disruption of the adipocyte lipid-binding protein (aP2 protein) gene impairs fat cell lipolysis and increases cellular fatty acid levels. J Lipid Res 40, 967-972.

29. Widjaja-Adhi MA, Lobo GP, Golczak M, et al. (2015) A genetic dissection of intestinal fat-soluble vitamin and carotenoid absorption. Hum Mol Genet 24, 3206-3219.

30. Beydoun MA, Nalls MA, Canas JA, et al. (2014) Gene polymorphisms and gene scores linked to low serum carotenoid status and their associations with metabolic disturbance and depressive symptoms in African-American adults. Br J Nutr 112, 992-1003.

31. Lobo GP, Amengual J, Li HN, et al. (2010) $\beta, \beta$-Carotene decreases peroxisome proliferator receptor $\gamma$ activity and reduces lipid storage capacity of adipocytes in a $\beta, \beta$-carotene oxygenase 1 -dependent manner. J Biol Chem 285, 27891-27899.

32. Amengual J, Gouranton E, van Helden YG, et al. (2011) $\beta$-Carotene reduces body adiposity of mice via BCMO1. PLOS ONE 6, e20644.

33. Garin-Shkolnik T, Rudich A, Hotamisligil GS, et al. (2014) FABP4 attenuates PPAR $\gamma$ and adipogenesis and is inversely correlated with PPAR $\gamma$ in adipose tissues. Diabetes 63, 900-911.

34. Kim G-S, Park HJ, Woo J-H, et al. (2012) Citrus aurantium flavonoids inhibit adipogenesis through the Akt signaling pathway in 3T3-L1 cells. BMC Complement Altern Med 12, 31.

35. Hsu C-L \& Yen G-C (2007) Effects of flavonoids and phenolic acids on the inhibition of adipogenesis in 3T3-L1 adipocytes. I Agric Food Chem 55, 8404-8410.

36. Lan H, Cheng CC, Kowalski TJ, et al. (2011) Small-molecule inhibitors of $\mathrm{FABP} 4 / 5$ ameliorate dyslipidemia but not insulin resistance in mice with diet-induced obesity. J Lipid Res 52, 646-656.

37. Furuhashi M \& Hotamisligil GS (2008) Fatty acid-binding proteins: role in metabolic diseases and potential as drug targets. Nat Rev Drug Discov 7, 489-503.

38. Boord JB, Fazio S \& Linton MF (2002) Cytoplasmic fatty acidbinding proteins: emerging roles in metabolism and atherosclerosis. Curr Opin Lipidol 13, 141-147.

39. Bagheri R, Qasim AN, Mehta NN, et al. (2010) Relation of plasma fatty acid binding proteins 4 and 5 with the metabolic syndrome, inflammation and coronary calcium in patients with type- 2 diabetes mellitus. Am I Cardiol 106, 1118-1123.

40. Xu A, Tso AW, Cheung BM, et al. (2007) Circulating adipocyte-fatty acid binding protein levels predict the development of the metabolic syndrome: a 5-year prospective study. Circulation 115, 1537-1543.

41. Ziouzenkova O \& Plutzky J (2008) Retinoid metabolism and nuclear receptor responses: new insights into coordinated regulation of the PPAR-RXR complex. FEBS Lett 582, 32-38.

42. Canas J, Lochrie A, Galena McGowan A, et al. (2014) Effect of a 2 -week intense life-style intervention followed by 6 -month carotenoid supplementation on fat depots, adiponectin and palmitoleate: a 6-month double blind placebo-controlled pilot study in obese children (645.6). FASEB J 28, Suppl. 1, 645.6.

43. Ruhl R, Bub A, Watzl B (2008) Modulation of plasma all-trans retinoic acid concentrations by the consumption of carotenoid-rich vegetables. Nutrition 24, 1224-1226. 